\title{
Editorial
}

\section{Clinical arrhythmology - an essential field of cardiology}

\author{
Mariana Floria \\ Guest Editor \\ "Sf. Spiridon" University Emergency Hospital, Iasi, Romania \\ "Grigore T. Popa" University of Medicine and Pharmacy, lasi, Romania
}

Clinical arrhythmology is an essential field for both electrophysiologists and cardiologists: in the case of the former to prevent the specialist from becoming a technician and in the case of the latter to fully understand the specialty. The clinical guidelines reaffirm this idea while also recommending an increasingly widespread interventional approach throughout the range of arrhythmias.

Arrhythmias occur early in life as a consequence of congenital constellations of anatomy and electrophysiology that change over time. There may be no structural cardiac abnormality (e.g., channelopathies like long or short QT syndrome or Brugada syndrome), a minimal structural abnormality (e.g., an accessory pathway causing Wolf Parkinson White syndrome) or a severe structural abnormality (e.g., ventricular septum defect with heart block). Later in life arrhythmias emerge as a consequence of acquired disease (e.g., ventricular tachycardia late after myocardial infarction due to the myocardial scar or other type of cardiomyopathy) or aging (atrial fibrillation). All these aspects of arrhythmias pathophysiology could be influenced by genetic susceptibilities.

Cardiovascular diseases are responsible for approximately 17 million deaths every year in the world, approximately $25 \%$ of which are sudden cardiac death [1].

Received: May 2017; Accepted after review: June 2017; Published: June 2017.

${ }^{*}$ Corresponding author: Mariana Floria, MD, PhD, "Grigore T. Popa" University of Medicine and Pharmacy, No. 16, Universitatii Street, 700115, lasi, Romania. E-mail: floria mariana@yahoo.com
Prevention of sudden cardiac death and management of patients with ventricular arrhythmias or syncope is one of the main purposes in arrhythmology [1]. Implantable cardioverter-defibrillator is the standard of care for prevention of sudden cardiac death in highrisk patients. Supraventricular arrhythmias are heterogeneous and, sometimes, very complex tachyarrhythmias for which new therapeutic interventions and sophisticated mapping and ablation tools were developed in view of curative treatment [2]. However these supraventricular tachycardia with narrow or wide QRS complex are rarely life threatening.

Arrhythmogenic right ventricular cardiomyopathy is a hereditary disease determined by progressive replacement of cardiac muscle fibers with a fibro-adipose tissue. This cause arrhythmia and sudden cardiac death. The review of Amalinei $C$ et al [3] presents an overview on traditional knowledge about this disease, adding updated information regarding molecular and genetic data.

Brugada syndrome is another inherited primary arrhythmia syndromes associated with malignant ventricular arrhythmias and sudden cardiac death [4]. The diagnostic electrocardiographic aspect could be unmasked in type 2 and 3 of Brugada syndrome with the class IC antiarrhythmic drug test. Implantable cardioverterdefibrillators are the most effective secondary prophylaxis therapeutic options for individuals with Brugada syndrome, but they might subject the patient to complications related to device implantation and inappropriate shocks [5]. Device pocket complications like infection and hematoma might rarely occur later after 
the implantation procedure due to senile pruritus [6]. Therefore device implantation could not be considered sometimes a benign intervention.

Long-QT syndrome is a congenital or acquired (drug-induced) disorder of the heart's electrical activity, inducing delayed repolarization that can cause also ventricular arrhythmias and sudden cardiac death4. The lack of a more specific algorithm to identify high-risk patients makes primary prevention troublesome [7]. However drug-induced arrhythmias are a challenge for clinicians from a wide range of specialties [7].

Long QT syndrome, Brugada syndrome and arrhythmogenic right ventricular cardiomyopathy known as inherited primary arrhythmia syndromes have a common mark, malignant ventricular arrhythmias, frequently manifested through syncope. Risk stratification in patients with these channelopathies and cardiomyopathies are still in debate. Heart Rhythm Society (HRS), the European Heart Rhythm Association (EHRA), and the Asia Pacific Heart Rhythm Society published recently a consensus regarding clinical guidance for diagnosis, risk stratification, and management of patients affected by inherited primary arrhythmia syndromes [4]. It is addressed to: individuals who survived a cardiac arrest at a young age (usually defined as $<40$ years) in the absence of a clinical diagnosis of cardiac disease, despite extensive clinical assessment; family members of individuals who died suddenly at young age with a negative autopsy; in patients and family members in whom the diagnosis of a channelopathy is clinically possible, likely, or established; and young patients with unexplained syncope [4]. For indications of genetic testing and also diagnostic, prognostic, and therapeutic implications of the results of genetic testing in patients affected by inherited arrhythmias (for the channelopathies and cardiomyopathies) and their family members there is another consensus statement [8]. Genetic study will increase in the future as the emphasis in rhythm disorders shifts to prevention.

Patient values and preferences for the management of cardiac tachyarrhythmias are important. When a patient has to make the decision whether or not to have a cardiac defibrillators implanted, patient preferences and values may vary depending on whether the indication is related to previous experience of life-threatening ventricular arrhythmias or sudden cardiac death (i.e. secondary prevention indication) or to a prophylactic indication (i.e. primary prevention indication) [9]. The same is valuable for antiarrhythmic drugs or ablation. Somewhere this is justified because there are still questions sans a right answer. For example the usefulness or suitability of defibrillation threshold testing the "to test or not to test" debate - still remains a matter of intense dispute among cardiologists [10]. Many centers worldwide are abandoning defibrillation threshold testing taking into account the balance between the clinical benefit and increased procedural risks [10].

Right ventricular apical pacing might have deleterious effects on cardiac structure and function [11, 12]. In these patients upgrading to biventricular pacing should be considered in patients requiring permanent or frequent right ventricular pacing for bradycardia, who have symptomatic heart failure and low left ventricular ejection fraction [12]. Upgrade to cardiac resynchronization therapy (with or without implantable cardiac defibrillator) not only improves the New York Heart Association functional class, but also causes reverse remodeling of the left ventricle with improved ejection fraction, similar to patients who undergo primary cardiac resynchronization therapy [11]. However the risk of complications is higher in upgrading procedures than in primary implantation procedures [11].

Wide QRS complex tachyarrhythmias are frequently a diagnostic challenge [13]. The presence of clinical symptoms or hemodynamic instability should not be used to distinguish ventricular tachycardia from supraventricular tachycardia [13]. Patients with dilated cardiomyopathy are exposed to ventricular arrhythmias (which are most frequently wide QRS complex tachyarrhythmias) and an increased risk of sudden cardiac death. In these cases the efficiency of antiarrhythmic drug therapy is limited and often the implantation of a cardiac defibrillator is required [14]. 
Electrical storm represents a lifethreatening condition characterized by electrical heart instability with several recurrent episodes of ventricular arrhythmias over a short period of time [15]. The treatment of electrical storm, this major clinical emergency, consists in prompt and complex therapeutical measures. In non-ischemic dilated cardiomyopathy unfortunately these are still not clearly defined [15].

There is no evidence that cardiac pacing prolongs survival in patients with sinus node dysfunction [12]. It seems that total survival and the risk of sudden cardiac death of patients with sick sinus syndrome (irrespective of symptoms) are similar to that of the general population [12]. In sick sinus syndrome when the patient's symptoms could be attributed to tachyarrythmic episodes rather to the bradycardia, the most efficient treatment option is to treat the patient's paroxysmal supraventricular tachycardia by radiofrequency ablation, rather than using cardiac pacing. Rarely a paroxysmal supraventricular tachycardia could coexist with sick sinus syndrome 16 . Therefore a clear cause-effect relationship between symptoms, which can be attributed to both the sinus node dysfunction

\section{References}

1. Priori SG, Blomström-Lundqvist $C$, Mazzanti $A$, et al. 2015 ESC Guidelines for the management of patients with ventricular arrhythmias and the prevention of sudden cardiac death. Eur Heart J 2015; 36(41):27932867.

2. Blomström-Lundqvist $C$, Scheinman $M M$, Aliot $E M$, et al. ACC/AHA/ESC guidelines for the management of patients with supraventricular arrhythmias--executive summary. a report of the American college of cardiology/American heart association task force on practice guidelines and the European society of cardiology committee for practice guidelines (writing committee to develop guidelines for the management of patients with supraventricular arrhythmias) developed in collaboration with NASPE-Heart Rhythm Society. J Am Coll Cardiol 2003; 42(8):1493-531.

3. Grigoras A, Knieling LA, Amalinei C. Arrhythmogenic right ventricular and the paroxysmal supraventricular tachycardia, is sometimes difficult to achieve [16].

The increasing prevalence of atrial fibrillation is a health challenge of the utmost importance. The Cox maze procedure, also known as a "cut-and-sew" technique, was first performed 30 years ago [17]. The principle of this technique is to create an electrical labyrinth (maze) of passages through which the sinoatrial node impulse finds a route to the atrioventricular node while preventing fibrillatory conduction. This first variant of this electrical labyrinth included lines for isolation of the posterior left atrium, a connection to the posterior mitral annulus, a cavo-tricuspid connection, a cavo-caval connection, and exclusion of the left atrial appendage. To improve the generation of transmural lesions, different endo-epicardial ablation strategies have recently been proposed [17]. In the last years some hybrid surgical ablation lesions sets were developed, usually in a manner less than the full Cox-Maze IV lesion set. This so called hybrid simultaneous ablation includes a "box lesion" aiming to isolate the pulmonary vein and the major ganglionated plexi [18].

cardiomyopathy: an overview and update. Arch Clin Cases 2017; 4(2):56-66.

4. Priori SG, Wilde AA, Horie $M$, et al. HRS/EHRA/APHRS expert consensus statement on the diagnosis and management of patients with inherited primary arrhythmia syndromes. Europace 2013; 15(10):1389-406.

5. Statescu C, Vasilcu T, Chiorescu IM, Tinica Gr, Arsenescu-Georgescu C, Sascau R. Optimal management of Brugada syndrome. Arch Clin Cases 2017; 4(2):67-73.

6. Ursaru $A M$, Ignat $A M$, Corduneanu $D$, Mazilu G, Petris AO, Tesloianu DN. Subpectoral pacemaker implant after repeated pocket complications due to "senile pruritus". Arch Clin Cases 2017; 4(2):74-81.

7. Marcu DTM, Hohaci A, Chiorescu MI, et al. Long-QT syndrome: to be or not to be iatrogenesis - a case report. Arch Clin Cases 2017; 4(2):82-87.

8. Ackerman MJ, Priori SG, Willems $S$, et al. HRS/EHRA expert consensus statement on the 
state of genetic testing for the channelopathies and cardiomyopathies: this document was developed as a partnership between the Heart Rhythm Society (HRS) and the European Heart Rhythm Association (EHRA). Europace 2011; 13:1077-1109.

9. Lane DA, Aguinaga L, Blomström-Lundqvist C, et al. Cardiac tachyarrhythmias and patient values and preferences for their management: the European Heart Rhythm Association (EHRA) consensus document endorsed by the Heart Rhythm Society (HRS), Asia Pacific Heart Rhythm Society (APHRS), and Sociedad Latinoamericana de Estimulación Cardíaca y Electrofisiología (SOLEACE). Europace 2015; 17(12):1747-1769.

10. Tesloianu DN, Ursaru AM, Ciudin RN. No Routine Defibrillation Threshold Testing (DFT) in all Implantable Cardioverter-Defibrilator's (ICD) recipients - Still a question? Arch Clin Cases 2017; 4(2):88-96.

11. Șuș I, Rudzik R, Dobreanu D. To pace or not to pace? Or how to pace? Arch Clin Cases 2017; 4(2):97-101.

12. ${ }^{* \star *} 2013$ ESC guidelines on cardiac pacing and cardiac resynchronization therapy: the task force on cardiac pacing and resynchronization therapy of the European Society of Cardiology
(ESC). Developed in collaboration with the European Heart Rhythm Association (EHRA). Europace 2013; 15(8):1070-118.

13. Chiorescu MI, Dabija E, Statescu C, Grecu M, Arsenescu-Georgescu C. Diagnosis and management of a wide QRS regular tachycardia. Arch Clin Cases 2017; 4(2):102109.

14. Bezim C, Ureche CG, Sascau RA, et al. Wide complex tachycardia - diagnostic and therapeutic challenges. Arch Clin Cases 2017; 4(2):110-115.

15. Mitu O, Balasanian M, Statescu $C$, et al. Complex management of electrical storm in a patient with non-ischemic dilated cardiomyopathy. Arch Clin Cases 2017; 4(2):116-123.

16. Ailoaei S, Tapoi L, Culica M, et al. Sick Sinus Syndrome - a case report of PSVT due to AVNRT. Arch Clin Cases 2017; 4(2):124-130.

17. Kirchhof $P$, Benussi $S$, Kotecha D, et al. 2016 ESC Guidelines for the management of atrial fibrillation developed in collaboration with EACTS. Eur Heart J 2016; 37: 2893-2962.

18. Floria M, Grecu M, Tinica Gr. Minim invasive epicardial posterior left atrial wall isolation: technique and results. Arch Clin Cases 2017; 4(2):131-138. 